\title{
Current Status of Research on Babesia Parasites in China
}

\author{
Ji Zheng1, Yiting Zhao1, Xiaoming Yang1, Jianfa Liu' ${ }^{*}$, Bin Xu${ }^{2 *}$, Wei Hu${ }^{3 *}$ \\ ${ }^{1}$ Medical School of Ningbo University, Zhejiang, China \\ ${ }^{2}$ Institute for Parasitic Diseases of Chinese Preventive and Control Center, Shanghai, China \\ ${ }^{3}$ School of Life Sciences of Fudan University, Shanghai, China \\ Email: *liujianfa@nbu.edu.cn, *xubin@npid.chinacdc.cn, ^huw@fudan.edu.cn
}

How to cite this paper: Zheng, J., Zhao, Y.T., Yang, X.M., Liu, J.F., Xu, B. and Hu, W. (2019) Current Status of Research on Babesia Parasites in China. Open Access Library Journal, 6: e5378.

https://doi.org/10.4236/oalib.1105378

Received: April 8, 2019

Accepted: April 26, 2019

Published: April 29, 2019

Copyright $\odot 2019$ by author(s) and Open Access Library Inc.

This work is licensed under the Creative Commons Attribution International License (CC BY 4.0).

http://creativecommons.org/licenses/by/4.0/

\begin{abstract}
Babesia is a kind of pear-shaped protozoa that is parasitic in mammalian red blood cells. It is mainly transmitted by sputum and blood transfusion. Babesia is a zoonotic parasitic disease with clinical manifestations similar to malaria. It has been found all over China. In many cases of Babesia infection, Babesia has caused great threat to humans and other mammals. However, there are few studies on Babesia in China. This paper will make a brief review on the morphology, life history and pathogenesis, epidemiology, diagnosis and treatment of Babesia in China.
\end{abstract}

\section{Subject Areas}

Infectious Diseases, Public Health

\section{Keywords}

Babesia, Research Status, China

\section{Introduction}

Babesia is a protozoan parasitic in mammalian red blood cells. It belongs to the genus Babesia. It was first discovered by the Romanian scientist Babes in 1888 from the diseased bovine red blood cells [1]. More than 100 species have been discovered since 1957. Yugoslavia reported the first case of human infection with Babesia [2]. Thousands of human infections have been reported around the world. With the development of diagnostic and detection techniques, cases of zoonotic Babesia have increased year by year in the America, Europe and new cases have been discovered in Asia and other places, and the disease has gradually become a worldwide human new parasitic disease.

${ }^{\star}$ Corresponding authors. 
It is currently recognized that four species of Babesia can infect humans, namely $B$. microti, $B$. divergens, $B$. duncani and $B$. venatorum. There are also three unidentified species of Babesia that are also infected with humans. They are CA strains, $\mathrm{MO}$ strains found in the United States, and $\mathrm{KO}$ strains similar to those found in Korea, B. ovine [3]. In China, Babesia infections are rare. In the past 30 years, only 10 cases have been reported in China [4]. However, the prevention and treatment of Babesia are urgent, and the domestic research on Babesia is still in the initial stage, this paper summarizes the research status of Babesia in recent years by summarizing the research reports on Babesia in terms of morphology, life history, pathogenesis, epidemiology, diagnosis and treatment of Babesia in China.

\section{The Morphology of Babesia}

The morphology of Babesia under light microscopy is very similar to that of Plasmodium falciparum. Its morphology is teardrop-shaped, ring-shaped, round, pear-shaped, rod-shaped or amoebic. The most typical is the Maltese cross, 4 pear-shaped. The tip of the merozoite usually forms a cross, which is a characteristic form of Babesia and an important morphological marker that distinguishes it from Plasmodium. According to the size of the worm, Babesia can be divided into two types: large worms and small worms. The large worms are $2.5-5.0 \mathrm{um}$ long, which exceeds the red blood cell radius. The small worms are $1.0-2.5 \mathrm{um}$ long and smaller than the red blood cell radius [5].

\section{The Life History of Babesia}

The life history of Babesia requires two hosts for ticks and vertebrates. In the sputum, completing gamete reproduction, followed by spore proliferation in the vertebrate body, it is parasitic in red blood cells, and the fissure is proliferated. Some merozoites form gametophytes, and the initial development of sexual reproduction begins, and then gradually develop into female and male gametophytes. Female and male gametophytes further develop into gametes in the scorpion, male and female gametes combine to form zygotes, and then develop into zygote, which can enter the blood and lymph of sputum, by replicating and invading various organs of sputum [6].

When an infectious biting host with mature sporozoites in the salivary gland sucks blood, the sporozoite enters the host with saliva, and the sporozoite enters the bloodstream and invades the red blood cells and develops into a trophozoite, which can be doubled in red blood cells for asexual reproduction. Form 2 or 4 worms. Individual or paired worms in red blood cells are often arranged at characteristic angles, with pointed ends being opposite. Finally, the red blood cells rupture, the worm body is released and can invade the new red blood cells and replicate, thus starting a new life history [7].

\section{The Disease Caused by Babesia}

The clinical manifestations of Babesiasis are similar to malaria. The main fea- 
tures of intermittent fever, splenomegaly, jaundice and hemolysis can cause renal dysfunction, and severe cases can cause death. As a new parasitic disease, the pathogenic mechanism of Babesia is gradually becoming a hot research topic. It can be confirmed that Babesia is parasitic on mammalian red blood cells. Studies have shown that rod-like proteins and micro-line apical membrane antigens play a crucial role in the invasion of red blood cells by Babesia [8] [9], which are secreted by rods and microfilaments, respectively, in which the microfilament secreted protein plays an adhesion role in the actin-myosin complex, which adheres to the host cell, allowing the worm to enter the host cell, and then The related lipids and proteins secreted by the rods work together to form nanoworm vacuoles, and the rod-shaped proteins play a key role in the invasion of the worms, and are the main virulence factors of the parasitic protozoa. At present, there are many pairs of Babesiabovis, Babesia ovine, Babesiagibsoni, Babesiabigemina, Oriental Babesia (Studies on RAP such as Babesiaorientalis), Babesiamicroti and Babesiamotasi-like [10].

\section{The Prevalence of Babesia}

Babesia is parasitic in mammalian red blood cells and is mainly transmitted by mites and bites. It can also be transmitted through blood transfusions and through the placenta. The host of Babesia is very extensive, and a variety of livestock and wildlife animals can be infected, becoming pathogen carriers and important sources of infection. Babesia is a zoonotic parasitic disease [11] [12].

Some rodents are important hosts of Babesia and play an important role in the long-term preservation, spread and prevalence of Babesia in nature. Rodents can infect voles, Babe, Baba, and Duncan. The squirrel, the squirrel, the yellow-breasted rat, the brown rat, the brown-backed rat, the black-lined squirrel, and the white-bellied rat can naturally infect the hamster Babe. Bovine subfamily is the natural host of many Babesia, a Babesia can be infected by a variety of cattle, and a cow can also infect a variety of Babe. Some sheep subfamilies can also be used as hosts for Babesia. In addition, canines, equines, and porcine animals can be infected with Babe [5]. Humans are also important hosts for Babesia, and the United States is the country that reports the most Babesia. The susceptible population of Babesia is the elderly, the history of spleen resection, the weakened immune system, the severe disease of the liver or kidney, and the farmers, herders and forestry workers who are often in contact with mites [13]. Most cases of infection with Babesia have a history of exposure to sputum. There are many kinds of aphids that transmit Babesia, mainly tiny calves, scorpion hard scorpion, long-horned blood scorpion, sulcus hard scorpion, blood red-headed scorpion scorpion, shoulder-shoulder hard scorpion, forest scorpion scorpion, grassland scorpion and scorpion Bloody. Different species transmit different Babesias. For example, tiny Burdock can transmit Babesia, Babesia and Mabba, and the scorpion can transmit Babesia, Babesia, Bigba beetle, hunter Babe, voles Babe and Babe [5]. 


\section{Diagnosis of Babesia}

\subsection{Microscopic Examination of Blood Smear}

A blood smear was prepared from the peripheral blood of the patient, and Giemsa staining or Wright's staining was used, and then the hamster Babesia worm was searched under a microscope. Under the light microscope, 1 - 6 worms can be seen in the red blood cells, and the size of the worms is generally $1.5-2.5 \mu \mathrm{m}$ [14]. The morphology of the larvae of Babesia is very similar to that of Plasmodium falciparum, and its shape is teardrop-shaped and ring-shaped. Shaped, round, pear-shaped, rod-shaped or amoeba-like, the most typical is the Maltese cross. However, due to the low detection rate of blood smears, the morphology and size of the early ring-shaped trophozoites of Babesia larvae are similar to those of erythrocytes in the erythrocytes such as Babesia and Plasmodium, so the diagnosis method is not very accurate [15].

\subsection{Animal Inoculation Separation Method}

The whole blood of the patient was inoculated into the hamster by the abdominal cavity (the hamster was a sensitive host of the hamster Babesia), and the peripheral blood smear was monitored for 6 weeks after the inoculation, and the pathogen appeared at about the second to fourth weeks. Animal vaccination method is sensitive, 300 pathogens can be found in about $1 \mathrm{ml}$ of blood, but this method is time-consuming and expensive, and is more suitable for retrospective diagnosis and auxiliary diagnosis, such as post-treatment review, long-term latent infection, etc., diagnosis [16].

\subsection{Indirect Fluorescent Antibody Test (IFAT)}

The basic principle of IFAT is to combine the unlabeled antigen (or antibody) for detection with the corresponding antibody (or antigen) in the sample to be tested to form an antigen-antibody complex, and then add fluorescein-labeled anti-human immunoglobulin or other The second antibody binds to form a fluorescent immune complex, which can display bright green fluorescence under a fluorescence microscope, thereby indirectly determining the antibody (or antigen) present in the sample to be tested. IFAT is currently the most widely used serological method for diagnosing Babesia in voles. The United States has applied it as a routine detection technique to the clinical diagnosis of voles Babesia [17] [18].

\subsection{Enzyme-Linked Immunosorbent Assay (ELISA)}

The basic principle of ELISA is to adsorb a known antigen or antibody on the surface of a solid phase carrier, so that the enzyme-labeled antigen-antibody binding reaction is carried out on the surface of the solid phase, and the unbound enzyme-labeled biomolecule is removed by washing, and then the coloring agent is added. Color, qualitative and quantitative analysis of the antibody or antigen are to be tested by the presence or absence of color reaction. However, 
the sera of the patient were detected by ELISA using the existing antigen. The results showed that Babesia and Plasmodium falciparum still had some cross-reaction, so that the specificity was low, and it was still necessary to find a more specific antigen for specific detection [14].

\subsection{Molecular Biology Diagnostic Technology (PCR)}

The outstanding advantages of molecular biology technology are high sensitivity and specificity, suitable for detection of insects with low infection rate, and are widely used in the detection of Babesia. Polymerase chain reaction (PCR) amplifies the target gene in large amounts, and then analyzes the amplified product by gel electrophoresis, sequencing analysis or probe hybridization. In addition, nested PCR, semi-nested PCR, real-time quantitative PCR, hot-start PCR, real-time fluorescent PCR and other derivative technologies developed on the basis of conventional PCR reactions further improve the sensitivity and specificity of detection [19]. The conventional PCR procedure was as follows: Babesia 18 s rRNA primer, upstream primer: 5'-AATACCCAA-TCCTGACA-CAGGG-3'; downstream primer: 5'-TTAAATACGA-ATGC-CAAC-3'. PCR reaction system 50 uL: pre-mixed Tapu enzyme $25 \mathrm{uL}, 10 \mathrm{umol} / \mathrm{L}$ upper and lower primers each 2.0 $\mathrm{uL}$, DNA template $10 \mathrm{uL}$, double distilled water $8 \mathrm{uL}$. PCR amplification conditions: $94^{\circ} \mathrm{C}$ for $5 \mathrm{~min} ; 94^{\circ} \mathrm{C}$ for $30 \mathrm{~s}, 60^{\circ} \mathrm{C}$ for $40 \mathrm{~s}, 72^{\circ} \mathrm{C}$ for $40 \mathrm{~s}$, a total of 35 cycles; $72^{\circ} \mathrm{C}$ for $5 \mathrm{~min} .5 \mathrm{uL}$ of the amplified product was spotted on $1.5 \%$ agarose, electrophoresed on a gel for $45 \mathrm{~min}$, and finally observed by a gel imaging system [20]. In addition, nested-PCR (nested-PCR) uses two pairs of primers in the inner and outer sides to perform two rounds of amplification reactions, so that the target fragment can be amplified from a small amount of template, which further improves the sensitivity of detection compared with ordinary PCR. Based on the product sequences amplified by the outer primers Bab1 and Bab4, Persing et al. designed the inner primers Bab2 and Bab3, increased the number of cycles to 50, and performed a second round of amplification under the same reaction conditions. It is $154 \mathrm{bp}$. Through two rounds of amplification, the sensitivity is increased to $3 \% \times 100 \%$ worm density, which is 10 times higher than that of ordinary PCR [21].

\section{Treatment of Babesia}

At present, the chemical drugs that control Babesia are mainly divided into two categories, which are directed against the medium hard palate or against Babesia itself. Commonly, therapeutic drugs used are clindamycin, quinine, atovaquone and azithromycin, but there are still no specific drugs, and there are also problems such as drug resistance, drug side effects, etc. Therefore, it is necessary to find alternative therapies and novel candidate vaccine molecules. RAP is an ideal candidate vaccine target and needs further study [10]. For people infected with Babesia, most asymptomatic people do not need treatment; those with symptoms should rest in bed, pay attention to nutrition; those with fever can be 
treated with physics and drugs, and those with anemia or hemolysis can give blood transfusion; in patients with renal failure, hemodialysis therapy is feasible, and transfusion therapy is feasible for critically ill patients [19]. The zoonosis caused by Babesia is second only to malaria, and research on the disease in China's parasitology community is on the rise. China looks forward to working with researchers around the world to face the challenges of the disease.

\section{Supported}

Financially supported by Zhejiang Scientific and technological programe (2017C37113).

\section{Conflicts of Interest}

The authors declare no conflicts of interest regarding the publication of this paper.

\section{References}

[1] Zhang, Q. (1982) Babesiosis of Human. Chinese Journal of Veterinary Science and Technology, 4, 63-64.

[2] Skrabalo, Z. and Deanovic, Z. (1957) Piroplasmosis in Man; Report of a Case. Document Med Gergr Trop, 9, 11-16.

[3] Zhou, J. (2016) Babesia Disease in Zoonosis. Proceedings of the Fifth Symposium of Veterinary Public Health Branch of China Animal Husbandry and Veterinary Society, 2.

[4] Ruan, W., Zhang, L., Chen, H., Lu, X., Zhang, X., Feng, Y. and Yao, L. (2017) Investigation on Natural Foci of Babesia in Central and Southern Zhejiang Province. Chinese Journal of Parasitology and Parasitic Diseases, 35, 125-129+135.

[5] Huang, T., Gao, Z. and Du, C. (2015) Advances in Research on Host Animal and Vector Epidemiology of Babesia. China Tropical Medicine, 15, 1151-1154.

[6] Chen, X., Li, X. and Wu, Z. (2012) Advances in Research on Babesia and Babesiosis. International Journal of Medical Parasitic Diseases, 39, 45-49.

[7] Hunfeld, K.P., Hildebrandt, A. and Gray, J.S. (2008) Babesiosis: Recent Insights into an Ancient Disease. International Journal for Parasitology, 38, 1219-1237. https://doi.org/10.1016/j.ijpara.2008.03.001

[8] Lobo, C.A., Rodriguez, M. and Cursino-Santos, J.R. (2012) Babesia and Red Cell Invasion. Current Opinion in Hematology, 19, 170-175. https://doi.org/10.1097/MOH.0b013e328352245a

[9] Straub, K.W., Cheng, S.J. and Sohn, C.S. (2009) Novel Components of the Apicom-Plexan Moving Junction Reveal Conserved and Coccidian Restricted Elements. Cellular Microbiology, 11, 590-603. https://doi.org/10.1111/j.1462-5822.2008.01276.x

[10] Yang, C., Cai, Y. and Chen, J.X. (2019) Advances in Research on Rhoptry Protein of Babesia. Chinese Journal of Schistosomiasis Control, 18, 1-5.

[11] Zhao, Y., Love, K.R., Hall, S.W., et al. (2009) A Fatal Case of Transfusion-Transmitted Babesiosis in the State of Delaware. Transfusion, 49, 2583-2587. https://doi.org/10.1111/j.1537-2995.2009.02454.x

[12] Sethi, S., Alcid, D., Kesarwala, H., et al. (2009) Probable Congenital Babesiosis in 
Infant, New Jersey, USA. Emerging Infectious Diseases, 15, 788-791. https://doi.org/10.3201/eid1505.070808

[13] Vannier, E., Gewurz, B.E. and Krause, P.J. (2008) Human Babesiosis. Infectious Disease Clinics of North America, 22, 469-488. https://doi.org/10.1016/j.idc.2008.03.010

[14] Zhang, J. and Chen, J. (2013) Advances in Diagnostic Techniques of Babesiosis. Chinese Journal of Zoonoses, 29, 714-718+723.

[15] Chen, S., Cai, Y., Chen, J., Lu, Y., Yu, Z., Zhang, Y., Ai, L. and Zhou, X. (2013) Ultrastructural Observation of Babesiamocroti. Chinese Journal of Zoonoses, 29, 1072-1075.

[16] Saito-Ito, A., Tsuji, M. and Wei, Q. (2000) Transfusion-Acquired, Autochthonous Human Babesiosis in Japan: Isolation of Babesiamicroti-Like Parasites with Hu-RBC-SCID Mice. Journal of Clinical Microbiology, 38, 4511-4516.

[17] Ma, O., Nishiguchi, K., Yuille, H.M., et al. (2011) Anti-HIV Siamycin I Directly Inhibits Autophosphorylation Activity of the Bacterial FsrC Quorum Sensor and Other ATP-Dependent Enzyme Activities. FEBS Letters, 585, 2660-2664.

https://doi.org/10.1016/j.febslet.2011.07.026

[18] Nakayama, J., Uemura, Y., Nishiguchi, K., et al. (2009) Ambuic Acid Inhibits the Biosynthesis of Cyclic Peptide Quormones in Gram-Positive Bacteria. Antimicrob Agents Chemother, 53, 580-586. https://doi.org/10.1128/AAC.00995-08

[19] Liang, Y. and Mu, L. (2016) Progress in Diagnosis and Treatment of Human Infection with Babesia. Inner Mongolia Medical Journal, 48, 1192-1195.

[20] Armstong, P.M., Katavolos, P., Caporale, D.A., et al. (1998) Diversity of Babesia Infecting Deer Ticks (Ixodesdammini). The American Journal of Tropical Medicine and Hygiene, 58, 739-742. https://doi.org/10.4269/ajtmh.1998.58.739

[21] Persing, D.H., Mathiesen, D., Marshall, W.F., et al. (1992) Detection of Babesiamicroti by Polymerase Chain Reaction. Journal of Clinical Microbiology, 30, 2097-2103. 